\title{
BASES TEÓRICAS DEL PROYECTO COMO INVESTIGACIÓN EN LA ENSEÑANZA UNIVERSITARIA DE ARQUITECTURA. PROPUESTA Y EJEMPLO DE IMPLEMENTACIÓN EN LA ESCUELA DE ARQUITECTURA DE LA ULA*
}

\author{
José Luis Chacón $R^{* *}$
}

Universidad de los Andes Mérida, Venezuela

Recibido: 29 julio 2013

Aprobado: 27 septiembre 2013
Proyecto de Carolina Salas.

Fuente: Reproducción fotográfica del autor.
Este artículo recoge un proceso de reflexión realizado al interno del área de investigación de la Universidad de los Andes ULA, sobre Teoría del Proyecto. En el mismo, se formulan las bases teóricas de una estrategia académica llamada Proyecto como investigación, la cual surge por una parte, de la comprensión originaria de la realidad universitaria, y por otra, de la implementación académica en una escuela -universitaria- de arquitectura de esta idea.

* Arquitecto de la Universidad de los Andes de Mérida, Venezuela, M.Sc. en Filosofía de la misma universidad, y Ph.D. en Composición Arquitectónica del Politécnico di Milano, Italia, 2009. Es Profesor Titular en la Facultad de Arquitectura y Diseño ULA, en Mérida, Venezuela. Coordina el grupo de investigaciones LISA_D, y la Maestría en Historia, Teoría y Crítica de Arquitectura, todos en la Universidad de Los Andes. Sus investigaciones principales son El Origen de la arquitectura, Trabajo de Ascenso, 1995; El espacio del ser, el ser del espacio. La noción de espacio en la Fenomenología de la Percepción de Maurice Merleau-Ponty, Tesis de Maestría, 200I II rapporto tra arte e architettura. Un "cerchio non chiuso", Tesis de Doctorado, 2009. Ha recibido reconocimientos por la Embajada de Canadá, el Salón Naciona de Arte Aragua, el Salón Arturo Michelena, la Universidad de Los Andes, y el Politecnico di Milano.

E-mail: jlchr@ula.ve

\section{RESUMEN}

La carrera de arquitectura en una universidad se realiza teniendo en perspectiva aprehender lo universal. En tal sentido, el "Proyecto como investigación" es una estrategia académica que permite la síntesis del conocimiento y lograr así el objetivo de la experiencia universitaria.

Esta propuesta consiste en el desarrollo de una investigación que coincide con el proyecto arquitectónico. Las bases teóricas de esta investigación proyectual son de orden epistemológico y metodológico. Por una parte, la epistemología del proceso de diseño indica que la idea y su proyección son las dos realidades que el mismo genera. Por otra parte, la metodología señala los dos modos de proceder en este proceso, por abstracción y por concreción. Los instrumentos que permiten desarrollar el Proyecto como investigación son el Libro de proyecto o portafolio, en el cual el dibujo y el texto se complementan con el fin de registrar el proceso.

Esta modalidad se ha implementado de manera experimental desde el $201 \mathrm{I}$ en la Escuela de Arquitectura de la Universidad de Los Andes arrojando resultados que han permitido que el trabajo de grado se convirtiera en una experiencia dinámica, creativa y universitaria.

\section{PALABRAS CLAVE}

Proyecto, Investigación, Arquitectura, Universidad. 


\section{THEORETICALBASIS FROMTHE PROJECT AS RESEARCH AT UNIVERSITY'S ARCHITECTURE TEACHING. SAMPLE PROPOSAL AND IMPLEMENTATION AT SCHOOL OF ARCHITECTURE}

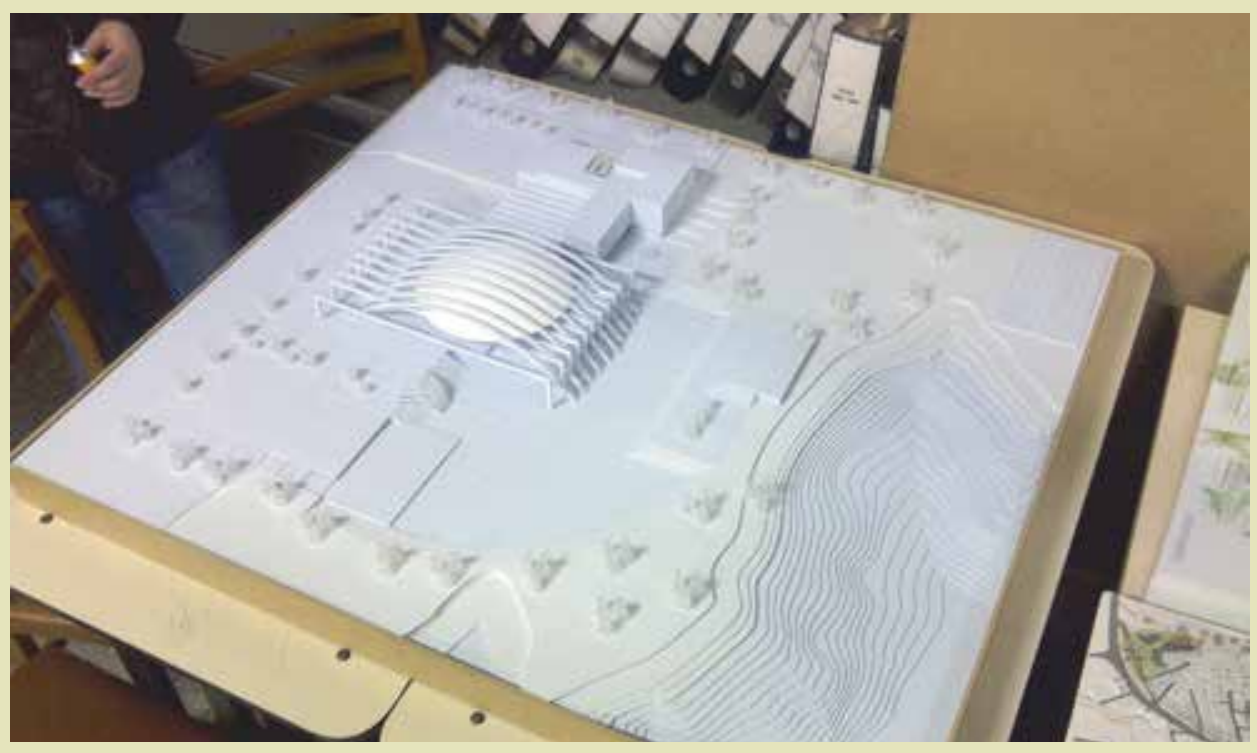

\section{ABSTRACT}

Architecture studies in university have the purpose of achieving universal knowledge. The "Project as research" is an academic strategy to do so by means of a Synthesis in which architecture becomes a full university experience. This proposal allows for a coincidence between research and project. On one hand, the epistemology of the design process points out that this project research produces two realities: the idea and its projection. On the other, its methology indicates the two procedures: by abstraction and by concretion. The instrument of the Project as research is the Project book or portfolio, in which drawing and writing form a unified registration of the process.

This strategy has been implemented as an experiment since $201 \mathrm{I}$ at the school of architecture of Universidad de Los Andes. Among the results was transforming the final project in a dynamic, creative and university experience.

\section{KEYWORDS}

Project, Research, Architecture, University.
Proyecto de Carolina Salas. Fuente: Reproducción fotográfica del autor. 


\section{INTRODUCCIÓN}

Este artículo está desarrollado a partir de la ponencia Hacer comprender el proyecto como investigación. La experiencia del TEGA en la ULA, presentada en el $5^{\circ}$ Seminario Internacional Arquitectonics Network', y recoge un proceso de reflexión realizado al interno del área de investigación sobre Teoría del Proyecto. En el mismo, se formulan las bases teóricas de una estrategia académica llamada "Proyecto como Investigación", la cual surge por una parte, de la comprensión originaria de la realidad universitaria, y por otra, de la implementación académica de esta idea en una Escuela -universitaria- de Arquitectura. La formulación de la propuesta no sigue propiamente una línea recta, que va de la teoría a la práctica, sino más bien una espiral que de la práctica va a la teoría y luego regresa otra vez a la práctica. En otras palabras, el "Proyecto como Investigación" es una propuesta que surge de una investigación creativa, análoga al proceso de diseño, en el cual teoría y práctica se fundamentan sintéticamente. La explicación de dicha propuesta sigue, sin embargo, una estructura lineal, con el fin de lograr una mejor comprensión, y así una divulgación efectiva que permita enriquecerla, tanto a nivel teórico como práctico, precisamente desde uno de los lugares clave en su proceso de generación.

\section{ARQUITECTURA Y UNIVERSIDAD}

En la mayoría de los Planes de Estudio de Arquitectura de las universidades del mundo se contempla la elaboración de un trabajo final, un Trabajo de $\mathrm{Grado}^{2}$ que se realiza al final de la carrera. Dicho trabajo tiene por lo general como fin confirmar la aprehensión de conocimientos logrados en estudios de arquitectura como requisito para obtener la titulación o certificación del grado final.

Este tipo de exigencia académica corresponde plenamente con los propósitos de una formación universitaria. La carrera de arquitectura en una universidad no busca meramente una capacitación profesional, ésta se realiza teniendo en perspectiva el horizonte del mundo; es desde ese lugar y momento, llamado universidad, que la pretensión milenaria -indiscutiblemente de base cristiana pero secularizada en el tiempo- de buscar lo uno en lo

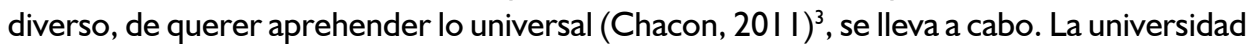
-recordemos- es una comunidad de intereses espirituales en búsqueda de la verdad ${ }^{4}$. Es por ello que está determinada por una actividad esencial: la investigación. Hurtado (2010) la define como un proceso metódico de búsqueda de conocimiento nuevo que procede según reglas, técnicas y métodos (p. 83). Buscar es, en breve, sinónimo de investigar. Esa búsqueda, sin embargo, ha sido interpretada de diversos modos generando diferentes visiones de universidad, como afirma Neira (2008); tal y como la universidad profesionalista, la educativa, la científica, aquella como factor de producción, y como factor de progreso (pp.2-5). En la universidad esa búsqueda, o investigación, esencialmente produce conocimiento; el conocimiento de lo uno en lo diverso. En efecto, afirmaba Villapalos (2007), "la

I Este congreso se llevó a cabo entre el 30 de Mayo de 2012 en Bucaramanga, Colombia.

2 En algunas escuelas lo llaman equivocadamente Tesis. Las implicaciones de una tesis son difícilmente aplicables a la arquitectura y su dinámica docente.

3 En dicho artículo se propone que la universidad actual debe ser fiel a su tradición milenaria, y por tanto, a lo inmanente en su nombre. "Universidad" es un término que se refiere tanto a unidad como a universo. Toda reflexión sobre la universidad, por consiguiente, debe hacer cuentas con esta inmanencia.

$4 \quad$ La Ley de Universidades de Venezuela actual resume la visión que tiene la Asociación de Universidades y que define la universidad como "búsqueda de la verdad". En Colombia la Ley 30 de 1992 regula la Educación Superior o universitaria sin definir explícitamente la universidad. Es el Artículo $4^{\circ}$ el que define la Educación Superior y lo hace de la siguiente manera: "La Educación Superior, sin perjuicio de los fines específicos de cada campo del saber, despertará en los educandos un espíritu reflexivo, orientado al logro de la autonomía personal, en un marco de libertad de pensamiento y de pluralismo ideológico que tenga en cuenta la universalidad de los saberes y la particularidad de las formas culturales existentes en el país. Por ello, la Educación Superior se desarrollará en un marco de libertades de enseñanza, de aprendizaje, de investigación y de cátedra". 
esencia de la Universidad hoy como hace casi un milenio es la de conservar, acrecentar, sistematizar y criticar el conocimiento" (p.26).

Por tanto, un trabajo de grado en arquitectura bajo esta consideración, sería la modalidad (espacial y temporal) para poder ${ }^{5}$ lograr el conocimiento universal-universitario. En las ciencias (puras, aplicadas y humanas) tal posibilidad se realiza tradicionalmente por medio de la tesis, una disertación intelectual de un tema de investigación que sintetiza el conocimiento y el cual viene comunicado principalmente a través del texto escrito ${ }^{6}$. Villapalos (2007) afirma en efecto que la "Síntesis del conocimiento" eso la misión principal de la universidad. La naturaleza de la arquitectura, así como la de las artes y del diseño, indica que dicha síntesis se logra pero por una vía diversa, ni textual ni numérica, sino por una correspondiente a su propia dinámica y especificidad. Particularmente esa vía es el proyecto, pensado sobre todo como investigación. El proyecto como investigación ${ }^{7}$ es pues una modalidad adecuada dentro de la experiencia universitaria para lograr la llamada "Síntesis del Conocimiento" en el campo de la arquitectura ( $y$ también en el arte y el diseño). En vez del texto escrito o del soporte de tablas numéricas, el dibujo y el modelado son los instrumentos primordiales para elaborar ese proyecto particular que desarrolla una temática con un fin confirmatorio. De allí pues la plena justificación de exigir que el trabajo de grado sea también en la forma de un proyecto, caracterizado por ser especial (por su condición sui generis), académico (por su contexto universitario) y efectivo (por su intención pedagógica).

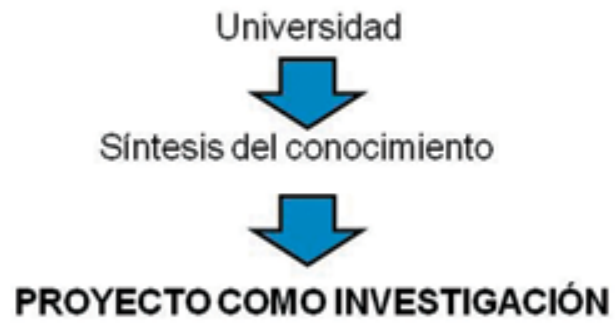

Investigación ---búsqueda sistemática

descripción, análisis, comparación conceptos y criterios

Proyecto - diseño arquitectónico

conceptualización, diseño, composición

situación y edificación

\section{El Proyecto como investigación 8}

Partiendo de la correspondencia entre universidad y arquitectura, se propone una estrategia académica llamada el "Proyecto como Investigación", que permite hacer arquitectura como parte integral de la experiencia universitaria. El "Proyecto como investigación" tal y
Figura I. Origen y propósito del Proyecto como investigación

Fuente: Elaborado por el autor. La universidad es el lugar de la libertad por excelencia y por tanto se utiliza el término "poder" porque es una posibilidad
que puede ser aprehendida según la libertad de cada quien.

6 A este respecto consultar el conocido libro Como se hace una tesis de Umberto Eco. Sin embargo, es de carácter técnico y no profundiza en las razones de la investigación.

7 El Proyecto como investigación es también el nombre de la propuesta y vendrá explicada a continuación.

8 Se toma prestado este nombre del título del $5^{\circ}$ Seminario Internacional Arquitectonics Network, "El Proyecto como Investigación, la Investigación como Proyecto", anteriormente mencionado. Dicho nombre coincide con el trabajo académico que se venía realizando y que se había implementado como Trabajo de Grado en la Escuela de arquitectura donde trabaja el autor. La originalidad del término reside pues en los organizadores del Seminario. 
como lo comprende Jaimes (2012) es una "comprensión del proyecto implícita al interés histórico-hermeneútico y emancipado del conocimiento", más allá del "interés técnico instrumental actual" (p.9) ${ }^{9}$. En este caso, el Proyecto como Investigación es en primer lugar, una modalidad para lograr la síntesis de los estudios de arquitectura en la culminación de la carrera universitaria. Consiste en plantear un proyecto arquitectónico que se desarrolle como proceso de investigación, sin que éste sea necesariamente una aplicación (de una cierta técnica), ni la fundamentación (de preceptos teóricos). Su objetivo es que la investigación coincida con el proyecto mismo. Por tanto, en el mismo se coloca en el proceso de generación de conocimiento (arquitectónico y universal) sin nunca dejar de lado la preponderancia de su condición proyectiva.

No obstante, tal idea puede conseguir resistencia en ambientes funcionalistas o formalistas de muchas escuelas de arquitectura, en las cuales el proyecto se concibe como actividad pragmática; puede en cambio conseguir mayor aceptación en escuelas de corriente conceptualista o de base humanista. En todo caso, esta propuesta pretende ser "amplia" e "integral", adecuada al pensamiento contemporáneo y sus exigencias, para ser implementada en escuelas que sean y se consideren verdaderamente universitarias ${ }^{10}$.

Ahora bien, el Proyecto como Investigación es una estrategia que se apoya en dos pilares teóricos, los cuales la sustentan y definen: uno es el pilar epistemológico y el otro es el metodológico. Ambas cuestiones conducen a su definición instrumental y, por tanto, a su implementación (que expondremos al final). Veamos entonces cada una de ellas en mayor detalle.

\section{Epistemología del proyecto (de lo proyectivo a lo proyectual)}

El proyecto arquitectónico se realiza por medio de un "proceso de diseño" ", que no es completamente pragmático, ni lógico, ni de inspiración artística; sino que es incluyente, paradójico y complejo. Según Chacón (2004), el proceso de diseño es en sí mismo una investigación, simultáneamente racional e irracional, heurística y referencial, teórica y práctica (p.26). Además, este proceso de investigación es sui generis en cuanto a generación y aplicación de conocimientos.

"El proyectista conoce y responde al mundo a través del proyecto", dice Spina (2004, p. I I). Su actividad, es decir, el diseño arquitectónico "es básicamente una disciplina intelectual de creación -como agrega Salas ( 1986) - cuyo centro de interés supone la materialización de la práctica arquitectónica" (p.4). Entre tantas reflexiones, éstas en particular ayudan a reconocer que el proceso de diseño es una sucesión de acciones que generan (y utilizan) conocimiento y que el mismo se caracteriza por ser productivo, compositivo, sintético y, sobre todo, creativo. Producir un proyecto es un acto creativo, pero diferente de la creación artística. ¿Cómo podríamos diferenciar una de la otra? En el diseño, la creatividad debe entenderse como investigación, creativa en efecto, pero que se concreta en el proyecto. El fin de dicha investigación es el proyecto, a diferencia de la creatividad artística cuyo objeto es la obra de arte. Por cuanto el proyecto es el objeto de investigación, ésta, además de creativa, es "proyectiva".

9 Este es un extracto del enunciado del Seminario. Jaimes fue el director junto con Josep Muntañola de dicho evento.

10 En este momento, la propuesta se encuentra aún en fase experimental en la FADULA (Facultad de Arquitectura y Diseño de la ULA).

II Este artículo hace referencia exclusivamente a investigaciones realizadas en la FADULA y que fueron publicadas principalmente en Cuadernos de Arquitectura de la FAAULA, No. I, 2004. Sin embargo, la formulación de la propuesta está basada en las reflexiones de autores de mayor universalidad, tales como Ludovico Quaroni, Gio Ponti, Peter Zumthor, entre otros. 
Según Hurtado (2010), la investigación es "proyectiva" cuando se elabora "una propuesta, un plan, un programa o un modelo, como solución a un problema o necesidad de tipo práctico, ya sea de un grupo social, de una institución, o de una región geográfica, en un área particular del conocimiento, a partir de un diagnóstico preciso de las necesidades del momento, los procesos explicativos o generadores involucrados y de las tendencias futuras, es decir, con base en los resultados de un proceso investigativo" (p.567). Esta definición tiene un amplio espectro de comprensión y aplicación en distintos campos, y para la arquitectura ( $y$ el diseño en general) es esencialmente pertinente. Pero es una definición reductiva; la idea "proyectiva" en este caso, para solucionar un problema o necesidad, sigue siendo aún pragmática.

Más que solución, el proyecto es una propuesta, algo que se propone en el presente pero que se refiere al futuro. Proyectar, de hecho, significa lanzar, dirigir hacia adelante, del latín proiectare, "lanzar adelante" 2 . El proyecto "lanza" una idea presente al futuro para su concreción. Aun cuando el proyecto puede ser un producto material de gran elaboración, es algo incompleto y logra su realización en el momento en que se convierte en hecho. Es por eso que el thelos de todo proyecto arquitectónico, por ejemplo, es la edificación.

Y ien qué consiste este proyecto arquitectónico? El proyecto de arquitectura es una idea dibujada (en planos y bocetos) y modelada (en maquetas y renders) que se propone de una edificación o urbanización para su futura construcción y eventual habitación (Chacón 2004). La clave está pues en la palabra idea. Como "Idea-Propuesta”, y no una solución a una necesidad, su confirmación no es inmediata; ésta se prolonga en el tiempo más allá del proceso de diseño. El proyecto por tanto se puede también entender como una hipótesis, sui generis por supuesto, que necesita luego ser confirmada en el momento de su construcción. Elaborar un proyecto arquitectónico consiste, en estos términos, en proyectar una idea.

\section{EPISTEMOLOGÍA}

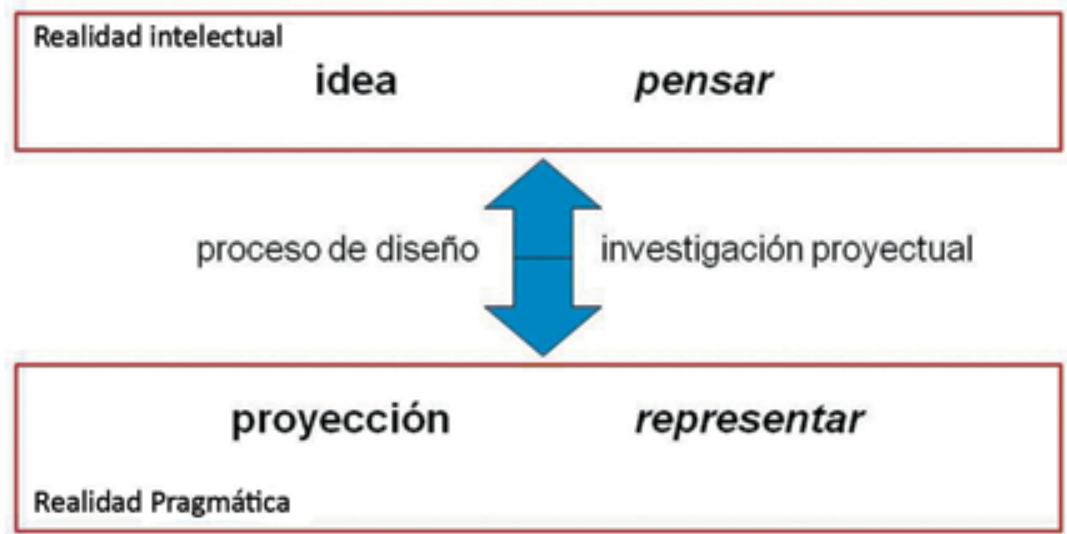

Siguiendo las consideraciones expuestas, si proyectar la idea-propuesta es producto de un proceso de investigación creativa-proyectiva, descubrimos en el fondo que su verdadera naturaleza es ante todo intelectual, desprendida completamente de nociones pragmáticas

12 Los componentes léxicos del término son el prefijo pro- (adelante) y lactare (lanzar frecuentemente). 
que la reducen. Las ideas, y en esto echamos mano de Platón, pertenecen a un mundo propio, el "mundo de las ideas", una realidad enteramente intelectual ${ }^{13}$. Lo eidético puede ser solo pensado, y su representación es en consecuencia "accidental".

Entonces, el proyecto comprende una doble realidad, integrada por la idea y su proyección; he aquí una curiosa equivalencia con la obra de arte. Proyectar una idea implica, en otras palabras, pensar y representar (Diez y Riega, 2004; Roncayolo, 1989). En tal sentido, el término proyectivo, cuyo sentido es esencialmente pragmático, no es suficiente para definir completamente esta actividad; el proceso de proyectar no es solamente proyectivo, es ante y sobre todo "proyectual". Es decir, es un proceso que se asimila a una investigación proyectual ${ }^{14}$ en la cual se piensa y se representa una idea. La noción de lo proyectual comprende precisamente esta doble realidad.

\section{Metodología de la investigación proyectual}

Entonces, icómo se logra el proyecto si el proceso de diseño se comprende como investigación proyectual? El conocimiento que se genera en tal situación sigue básicamente dos caminos: de forma deductiva o de forma inductiva. Siguiendo la teoría del conocimiento, la deducción es aquel proceso que parte de lo ideal y emprende un camino para desglosar, explicar y dar razón del mismo. La inducción, en cambio, parte del particular que se encuentra en la realidad, reúne, coordina y sintetiza para alcanzar algo en un estado superior que une los particulares.

Salas (1986), en relación al proceso de diseño, habla de un proceso que va de lo abstracto (la primera fase que llama conceptualización creativa) a lo concreto (la última fase, concreción creativa). Según esta concepción, el inicio de este proceso es algo general y abstracto, y el final específico y concreto; las ideas, entendidas como intenciones mentales, pertenecen al primer ámbito mientras que el proyecto, entendido como expediente gráfico, al segundo. En este caso, el proceso sigue un camino deductivo.

Pero cuando el proceso de diseño se concibe como investigación, como se propone, esta unidireccionalidad se desdibuja. El proceso de investigación puede partir de la elaboración de un expediente de planos y maquetas (proyecto), cuyo objetivo es una profundización referida al contenido temático $\left(\right.$ tema $\left.^{15}\right)$ inmanente en el proyecto. Pero puede también partir de ideas abstractas (temas) que pretenden concretarse en un expediente proyectivo (proyecto). En ambos casos se presentan metodologías completamente distintas; una inductiva y la otra deductiva, coincidiendo con lo planteado por la teoría del conocimiento.

En consecuencia a lo anteriormente expuesto, la propuesta del Proyecto como Investigación presenta dos alternativas metodológicas, familiares al proceso de diseño, que coinciden precisamente con las cuestiones epistemológicas expuestas. Una se denomina la vía concreta, que sigue el sentido del representar al pensar, es decir, de la proyección (o de la intención proyectiva) a la idea (de la investigación proyectual); esto traza un camino metodológicamente inductivo. Y la otra, la vía abstracta, que va de la idea (proyectual) a la proyección (intención proyectiva), por el camino metodológico de la deducción, en el sentido de la pensar al representar. Por medio de cualquiera de estas vías, en definitiva, el investigador-proyectista despliega de manera cabal la investigación proyectual.

3 Aunque la Teoría de las Ideas de Platón no está formulada en una sola obra, una definición general se encuentra en Fedón, o sobre el alma.

I4 Se utiliza el término "proyectual" con el fin de diferenciarlo de "proyectivo", usado por Hurtado (20I0).

15 En pasadas reflexiones, se ha utilizado el término "tema" para indicar la condición eidética del proceso de diseño. 
En el ámbito docente esto se puede entender de la siguiente forma: si el estudiante (a final de carrera) quiere explorar y desarrollar como trabajo de grado un proyecto arquitectónico de una escuela, de un hotel, de un edificio residencial, entre otros, y para que éste sea una investigación proyectual, de esa intención proyectiva debe descubrirse y resaltarse una idea proyectual, un tema particular a investigar, por ejemplo en referencia a la escuela, una filosofía educativa particular, la problemática social en las aulas, la relación entre varias tipologías de escuelas, etc.; y así sucesivamente con los otros casos. La idea proyectual es, por tanto, de carácter general y engloba la representación proyectiva. Si en cambio, el estudiante tiene en mente temas como la percepción, la intervención del patrimonio, la estética contemporánea, etc., es decir una idea proyectual, la investigación conlleva a un expediente proyectivo que resulte de la temática misma ${ }^{16}$, como puede ser un centro comercial, una vivienda, un complejo deportivo, entre otros. La escogencia de lo proyectivo está pues íntimamente ligada con lo proyectual y viceversa.

Ambas modalidades generan conocimiento de formas completamente diferentes. Paradójicamente la inducción, siendo la modalidad más exigente a nivel intelectual, es la más aceptada en las escuelas de arquitectura y subsiste como práctica institucionalizada. El particular es siempre lo más evidente en lo cotidiano; es más fácil afrontar una escuela que una ideología pedagógica. En cambio, la deducción, procedimiento lógico que está a la base de nuestra educación positivista, resulta más compleja y menos divulgada en las escuelas de enseñanza arquitectónica. Partir de ideas para llegar a su representación concreta es una estrategia propia de las artes pero peligrosa en el campo arquitectónico porque puede desencadenar en abstracciones sin concreción ${ }^{17}$. En todo caso, esta propuesta pretende dar cabida a modos diversos de asumir la proyección arquitectónica, siempre y cuando se entienda que se busca alcanzar un estadio intelectual mayor que supere la condición pragmática y tecnicista del mero oficio.

\section{METOdOLOGÍA}

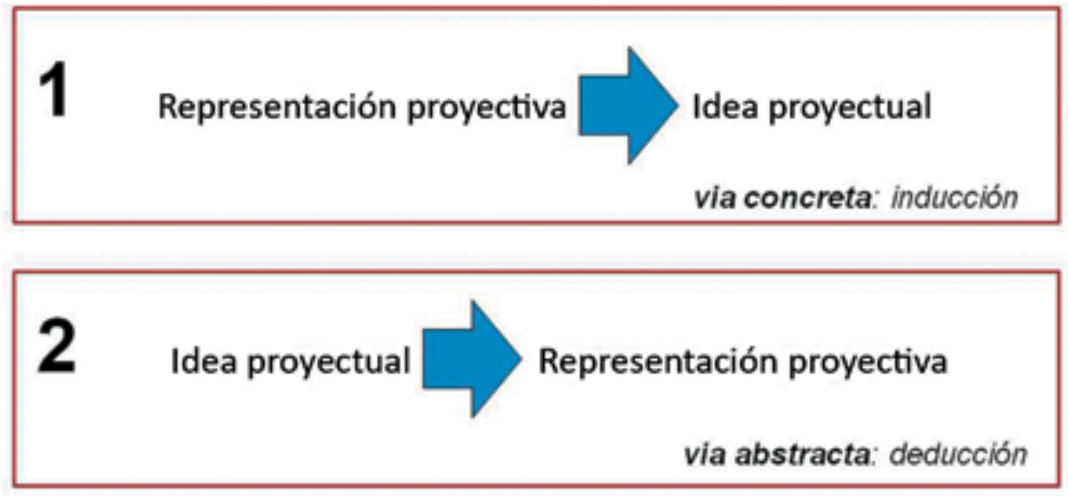

\section{Paréntesis histórico}

Históricamente en arquitectura, la epistemología y metodología del proceso de diseño se caracteriza por tener tres grandes momentos o etapas. El de Beaux Arts, el cual parte de la tipología y se desarrolla según un procedimiento figurativo (de mímesis) para lograr

16 Algunos sostendrán que el proyecto debe demostrar o confirmar su relación con el tema, pero esta es una visión demasiado positivista y puede limitar en vez de posibilitar.

17 Como sucede en muchas escuelas de corte conceptualista. 
una composición. El del Movimiento Moderno, que desde la geometría pura procede por abstracción para obtener igualmente una composición de carácter preponderantemente formal ${ }^{18}$. Y el del Conceptualismo, el cual surge desde los conceptos (ideas) y resulta en una especie de poiésis abstracta.

Se puede decir entonces que estas etapas se diferencian en dos categorías distintas: la primera, epistemológicamente inductiva, y operativamente (metodológicamente) concreta; y las dos últimas que en cambio son epistemológicamente deductivas y operativamente abstractas.

\section{INSTRUMENTOS DEL PROYECTO COMO INVESTIGACIÓN}

La tentación más grave de una investigación proyectual es pretender realizar un proyecto arquitectónico fundamentado teóricamente. Esto indica una división en las actividades, una fase "teórica" y otra propiamente "proyectiva". El Proyecto como investigación es, de hecho, un sólo proceso continuo, en el cual la investigación coincide con el proyecto, como se afirmó anteriormente. En este sentido, es análogo al proceso de creación artística; en una obra de arte el proceso se confunde con el resultado final, y llegan a ser indisolublemente equivalentes.

Para evitar la escisión entre teoría y praxis, el Proyecto como Investigación solicita un "Libro de Proyecto" como instrumento básico de trabajo, independientemente de la vía que se tome, sea la concreta o la abstracta. Basado en los cuadernos de estudio (de por ejemplo, Le Corbusier, Louis Kahn, o Aldo Rossi, y en sintonía con los libros de artista ${ }^{19}$, dicho libro se comprende como el registro, o memoria, del proceso del proyecto. Como tal, este libro recoge toda la información al igual que toda la reflexión acerca de la investigación. Es un instrumento de trabajo vital que va creciendo y mutando en el tiempo, análogo a la dinámica intrínseca de un palimpsesto. Al final del proceso, después del resultado logrado, este libro se convierte en un producto equivalente al expediente proyectivo, necesario incluso para validar (científicamente) la investigación.

Por cuanto el término "Libro de proyecto" no es común en el campo de la arquitectura, éste se puede relacionar con otro más conocido: el "portafolio", pero entendido en este caso como "portafolio proyectual". Esta noción tiene una acepción tanto en la realidad profesional, el portafolio como recolección de productos terminados, como en el ámbito académico, es decir, como registro del proceso de investigación. Tal y como dice Fernández (2004), el portafolio -en el ámbito académico- "responde a dos aspectos esenciales del proceso de enseñanza-aprendizaje, implica toda una metodología de trabajo y de estrategias didácticas en la interacción entre docente y discente". A lo que agrega, "es un método de evaluación que permite unir y coordinar un conjunto de evidencias para emitir una valoración lo más ajustada a la realidad que es difícil de adquirir con otros instrumentos de evaluación más tradicionales que aportan una visión más fragmentada" (p.|3I). Para el Proyecto como investigación el portafolio, como sinónimo a Libro de Proyecto, recoge, ordena y procesa el trabajo no terminado de apuntes, bocetos y modelos del proceso de investigación proyectual hasta su culminación.

La introducción de un instrumento como el Libro de Proyecto no representa ninguna novedad en el campo de la arquitectura (o del diseño y las artes), pero en la era digital y

18 El Funcionalismo puede ser considerado una consecuencia del abstraccionismo geométrico del Movimiento Moderno, siendo los esquemas funcionales el fundamento que sustituye el juego de formas geométricas.

19 Sintéticamente, el Libro de artista, según Nelson Gómez Callejas (s.f.), es "un soporte para el acontecimiento estético". 
tecnocrática es una metodología contracorriente. En el presente, el dibujo sobre soporte material y como instrumento de conocimiento, parece ocupar un lugar secundario. Por ello, el Proyecto como Investigación lo repropone, no como una estrategia restauradora sino como metodología originaria del oficio mismo.

En sintonía con los ejemplos de los grandes maestros, el dibujo es la base de la investigación (en arte y arquitectura). La "búsqueda paciente" que señalaba Le Corbusier se hace principalmente por medio del dibujo. Entonces, el énfasis es en lo gráfico, en el esquema, el dibujo, el boceto, el modelo, en combinación con el texto escrito, el cual sigue teniendo su importancia.
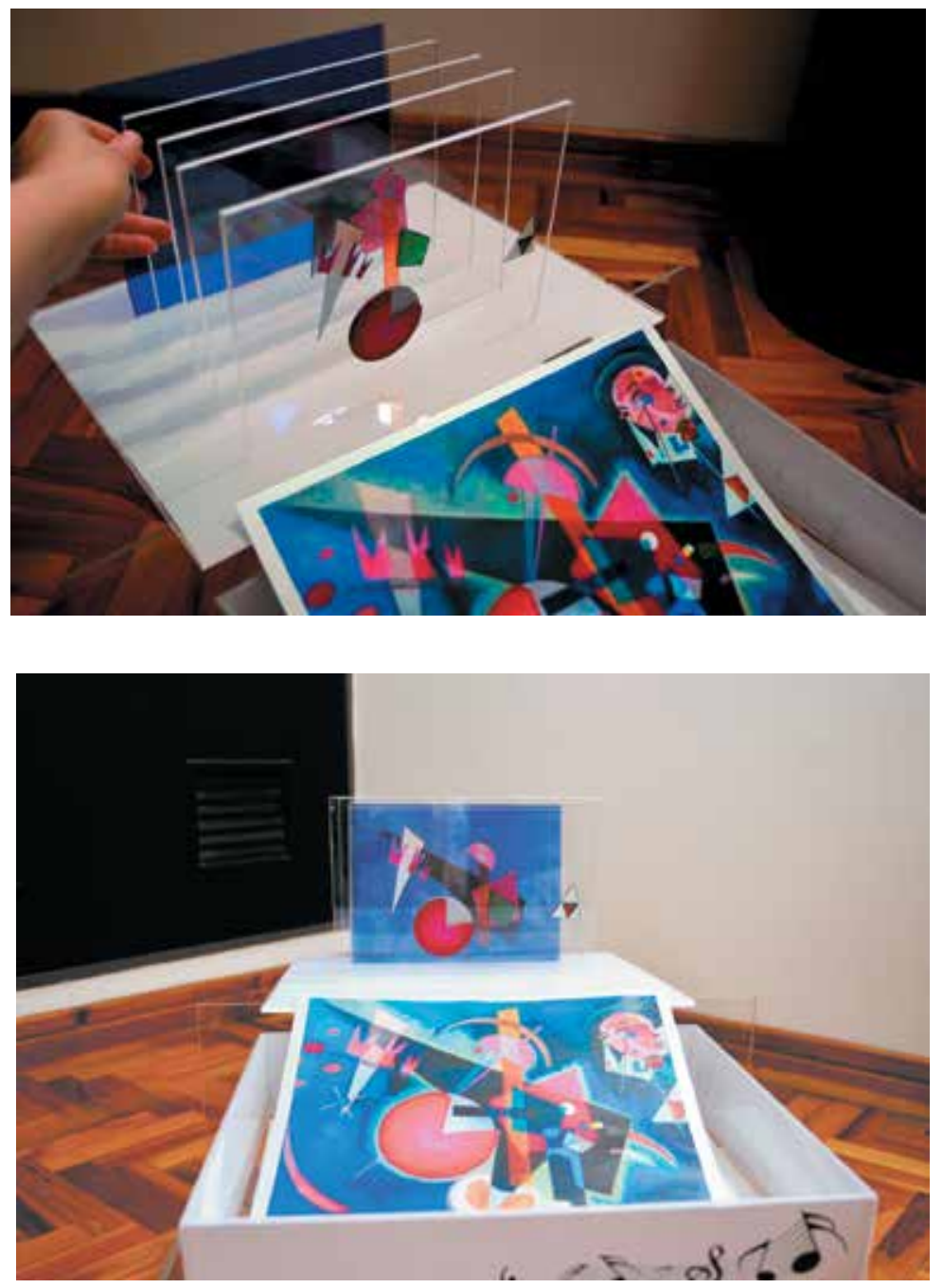

Pareciera que el riesgo que se corre con éste puede ser la pérdida del uso del lenguaje escrito. Aun cuando las experiencias del libro de artista son un punto de referencia, la comprensión de la relación entre grafismo y literatura es fundamental (así como sucede con la relación entre proyecto y construcción) para no dejar de lado la preponderancia de la comunicación lingüística. Basta con revisar los libros de Le Corbusier para comprender esta relación.
Figura 4. Ejemplo de un Libro de proyecto Fuente: Fotografías del autor. 


\section{La experiencia del TEGA ${ }^{20}$ en la Escuela de Arquitectura de la ULA}

De acuerdo con la normativa que regula el TEGA, éste se define como "el ejercicio concluyente del proceso enseñanza-aprendizaje del eje Taller de Diseño Arquitectónico, en el cual el alumno elabora un trabajo de investigación de libre elección enmarcado en las dimensiones funcionales, formales, tecnológicas, históricas y ambientales de la arquitectura, cuyo resultado es un proyecto arquitectónico"21 (FADULA, 2006).

Desde su implementación al inicio de la década de los noventa ${ }^{22}$, esta modalidad ha generado grandes contradicciones en su comprensión y aplicación. En el tiempo el TEGA ha generado algunos logros sobresalientes, pero los conflictos internos lo han reducido lamentablemente a mero requisito administrativo ${ }^{23}$.

Afortunadamente, a pesar de su devenir accidentado, el TEGA ha mantenido su definición original: un trabajo de investigación en arquitectura con base en el proyecto; en pocas palabras, una investigación proyectual. Entre los resultados sobresalientes se evidencia cómo esa pretendida correspondencia entre investigación y proyecto es posible.

A partir de 2010, bajo la coordinación del presente autor, se implementó un cambio en el modo de concebir y guiar el TEGA, reorientándolo hacia la concepción del Proyecto como investigación. La Nueva Modalidad ${ }^{24}$ se implementó de manera experimental en 201 I con el Grupo 35 del TEGA. En esta modalidad participaron 19 estudiantes quienes decidieron hacerlo en conjunto con sus tutores; debía existir un conocimiento y un convencimiento por la nueva propuesta para que la experiencia pudiese fluir. La realizaron a lo largo de los dos semestres estipulados más la prórroga de ocho semanas que se les concede. Tanto los estudiantes como los profesores (tutores) evaluaron la experiencia de manera positiva. Sin embargo, la modalidad introdujo una serie de complicaciones de tipo administrativo que generó descontento en el Departamento de Composición Arquitectónica.

Figura 5. El TEGA y el Proyecto como Investigación

Fuente: Elaborado por el autor

\section{TEGA: El proyecto como investigación}

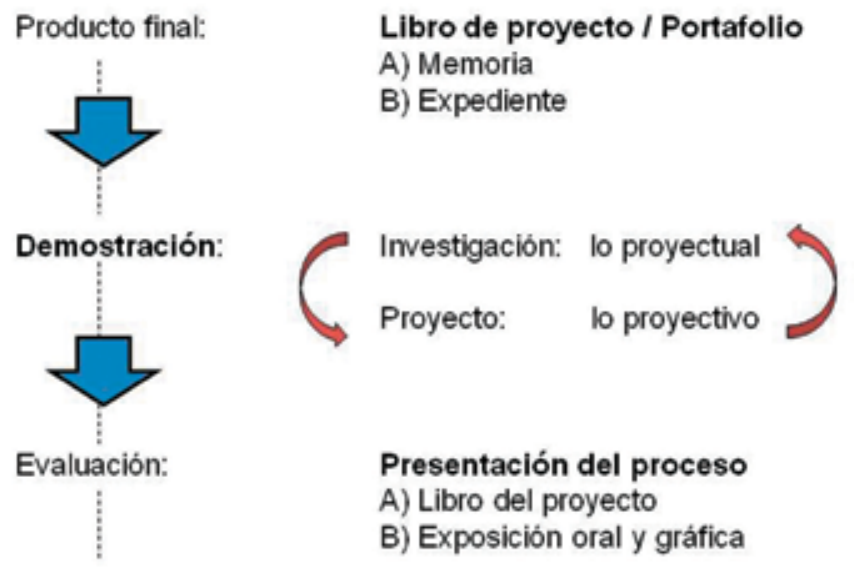

\footnotetext{
20 Trabajo Especial de Grado en Arquitectura.

21 Normas para el Trabajo Especial de Grado de la Escuela de Arquitectura.

22 En 1987 se aprueba un nuevo Plan de Estudios que se implementa por fases. La última fase fue precisamente la del TEGA que se puso en marcha en 1992.

23 En 2002, el Dpto. de Composición Arquitectónica elabora los Programas Marco para los Talleres de Diseño Arquitectónico, en donde se comprendía de manera integral el TEGA. En el 2006 la Comisión Técnica elabora un reglamento definitivo.

24 Esta fue la denominación dada al nuevo procedimiento.
} 


\section{Estos son los puntos más resaltantes del resultado de la Nueva Modalidad:}

I. Esta modalidad devolvió al TEGA la dinámica discursiva en la evaluación de arquitectura. La investigación proyectual se presenta como discurso que necesita la confrontación para que los alcances logrados sean plenamente comprendidos.

2. La presentación del trabajo se volvió más creativa y no un mero acto burocrático. Se lograron modos alternativos e interesantes de presentar el proyecto y lo que lo sustenta. El Libro de proyecto se convierte así en la memoria gráfica y creativa del proceso de investigación proyectual.

3. Se permitió comprobar la importancia de concebir el Proyecto como Investigación. No desde una posición profesional o pragmática, sino desde una perspectiva académica, en la que la arquitectura se transforma en una experiencia universitaria de verdadera síntesis de conocimiento.

Desafortunadamente esta experiencia no ha sido continuada por los coordinadores sucesivos, salvo a nivel reducido ${ }^{25}$. La Escuela de Arquitectura de la ULA es en esencia de corte funcionalista, donde la arquitectura es considerada un hecho pragmático. Su vocación por la investigación ha estado limitada a los campos de la historia, de la tecnología y recientemente del ambiente; mientras que en el ámbito del diseño arquitectónico ${ }^{26}$ ha sido muy escasa. Una propuesta que conciba la arquitectura como actividad cognoscitiva, tal como lo piensa el Proyecto como investigación, implica un cambio de conciencia en el reconocimiento de la especificidad de la arquitectura y su enseñanza dentro de la realidad universitaria. Esta propuesta pretende ser, no obstante, un paso en esa dirección.
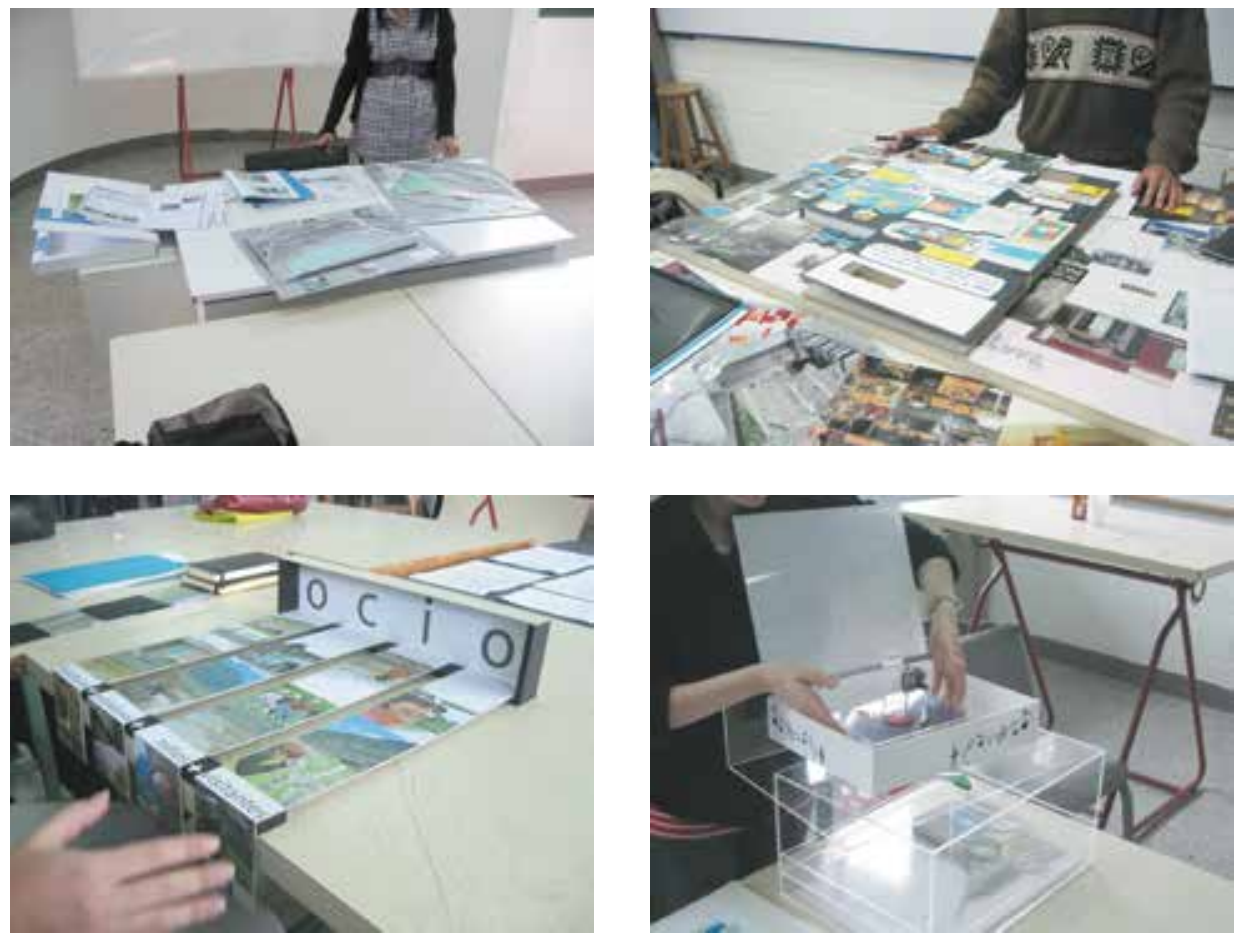

Figuras 6a- 6b- 6c- 6d. Presentación de Libros de proyecto del grupo de estudiantes del TEGA. Fuente: Fotografías del autor.
25 Ésta continúa aún como modalidad experimental solo con los grupos tutorados por el autor

26 En esta escuela el proyecto arquitectónico se enseña en los Talleres de Diseño Arquitectónico a lo largo de los 10 semestres de la carrera. Sus profesores están adscritos administrativamente al Departamento de Composición Arquitectónica. 
Figura 7. Proyecto de Carolina Salas Fuente: Fotografías del autor.

\section{ANALOGÍA}

SOTO FORMAL

(GEOMETRIA RACIONAL)
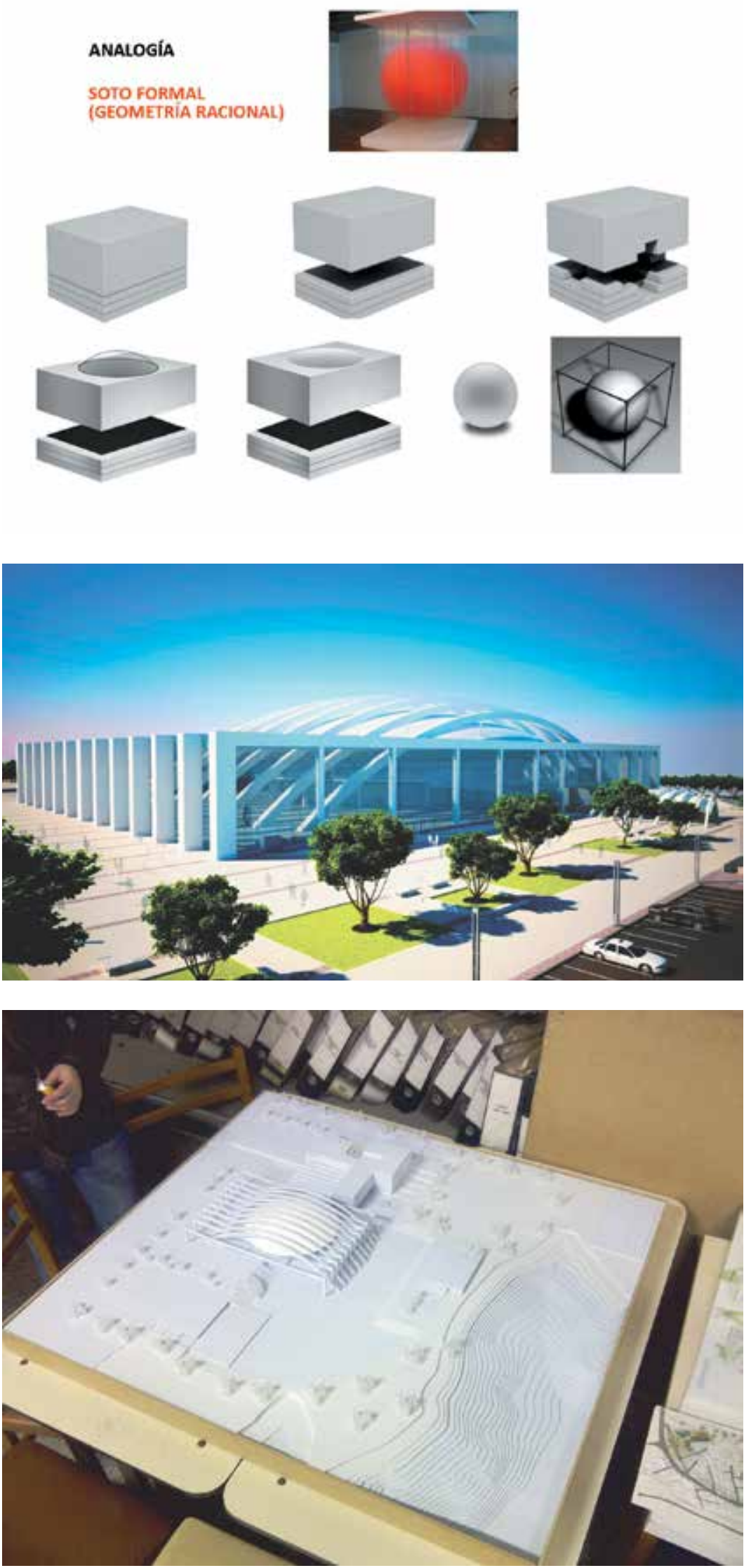

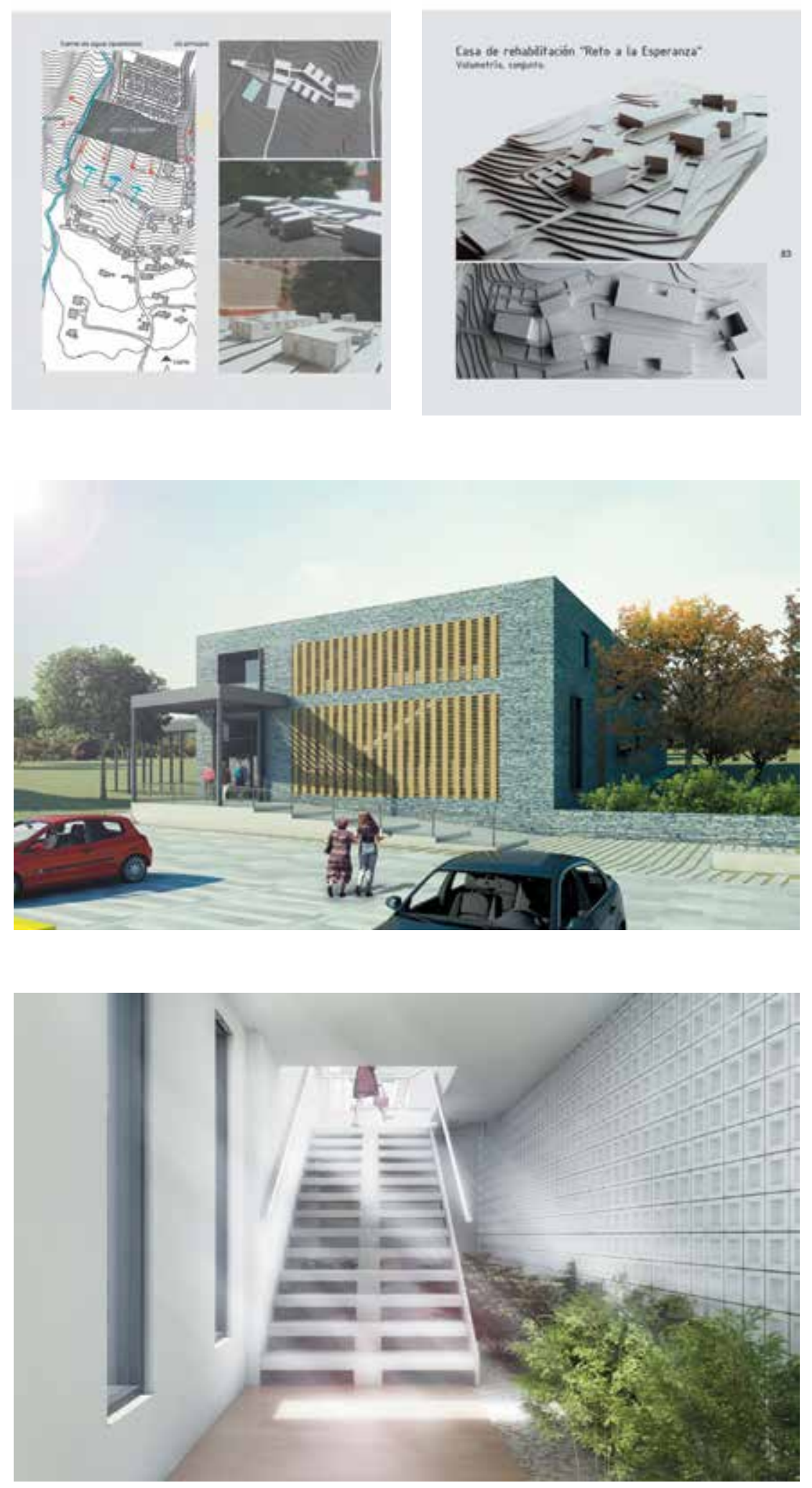

M 19 REVISTA M VOL. 10 No.2. JULIO-DICIEMBRE 2013 - FACULTAD DE ARQUITECTURA • UNIVERSIDAD SANTO TOMÁs COLOMBIA 

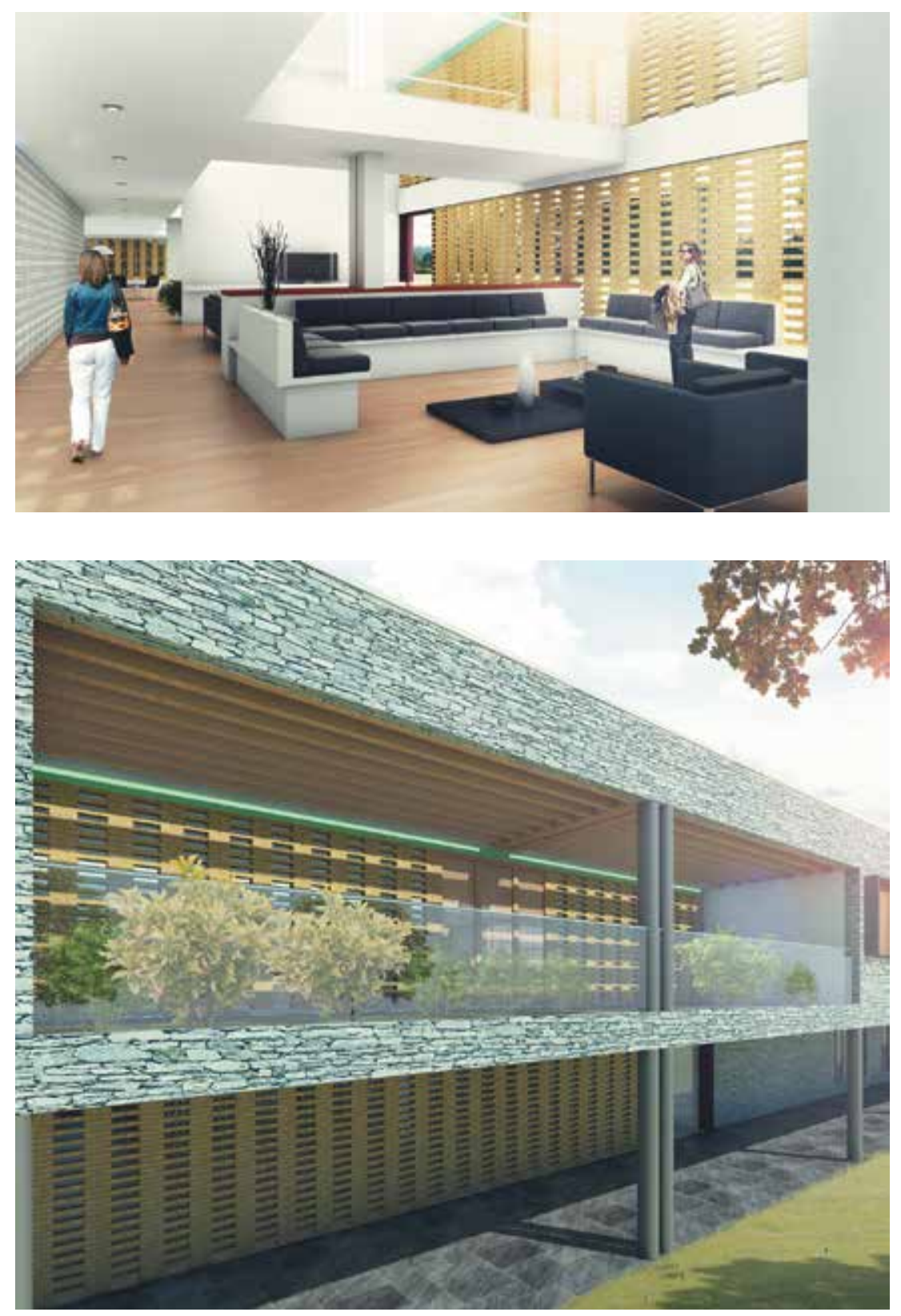

\section{CONCLUSIONES}

Si se comprende la experiencia del TEGA como implementación del Proyecto como investigación, la misma resulta ser aún limitada para confirmar los alcances de la propuesta. Pero precisamente por la dinámica de la cual ésta ha surgido, la experiencia viene a ser fuente de su origen y destino de su desarrollo. Para la comprensión es necesario que la teoría preceda a la práctica, pero para la experiencia, la teoría surge de la práctica, pensada, y vuelve a ella para enriquecer a ambas. La investigación en arquitectura, al igual que en el arte, no sigue los caminos de las ciencias puras o aplicadas. El peso del hacer es fundamental; el hacer es ya pensar.

Por consiguiente, la propuesta del Proyecto como investigación no pretende ser una mera teoría o una teorización del proceso proyectivo. Es una estrategia académica que necesita 
enriquecerse todavía aún más, con la reflexión teórica y con mayor práctica, dentro del recinto universitario. Sería deseable pues obtener un intercambio de diversas observaciones o incluso de posibilidades de aplicación. De esta manera, esta propuesta, como todo proyecto, queda abierta a ser mejorada en la medida que se vaya desarrollando y construyendo, con el fin, eso sí, de hacer de la arquitectura y su enseñanza un oficio plenamente universal.

\section{REFERENCIAS}

Chacón, J.L. (20I I). Ante la nueva Ley de universidades. Artículo inédito.

Chacón, J.L. (2004). El Proceso de Diseño Arquitectónico. Guía para estudiantes de Taller de Diseño Arquitectónico, en Cuadernos de Arquitectura de la FAAULA, ( I ). Mérida: FAAULA.

Diez y Riega, O. (2004). Cómo aprender a pensar para diseñar, en Cuadernos de Arquitectura de la FAAULA, (I). Mérida: FAAULA.

FADULA. (2006). Normas para el Trabajo Especial de Grado de la Escuela de Arquitectura. Mérida: FADULA.

Fernández March, A. (2004). El portafolio docente como estrategia formativa y de desarrollo profesional, en Educar, (33).

Gómez Callejas, N. (s.f). El libro de artista. Consultado en junio 20 de 2013 . Disponible en: http://webdelprofesor.ula.ve/arte/callejas

Hurtado de Barrera, J. (2010). Metodología de la investigación, una comprensión holística. Caracas: Ediciones Quirón-Sypal.

Jaimes Boitía, S. (2012). El Proyecto como investigación, la Investigación como proyecto, en Memorias del Seminario Internacional Arquitectonics Network en América "El Proyecto como investigación, la Investigación como proyecto”, Bucaramanga.

Neira, E. (2008). Universidad-Autonomía-Cogobierno. Disponible en: http://www.saber.ula. ve/handle/I 23456789/I5673. Consultado el 20-06-I3.

Roncayolo, D. (1989). La Obra y el Oficio, Trabajo de Ascenso. Mérida: Universidad de Los Andes.

Salas, J de D. (1986). La Enseñanza del Diseño Arquitectónico, entre lo concreto y lo abstracto. Mérida: Facultad de Arquitectura ULA.

Spina, P. (2004). Enfocando la actividad: El diseñar, en Cuadernos de Arquitectura de la FAAULA, (I). Mérida: FAAULA.

Villapalos Salas, G. (2007). La Universidad Universal, en $A B C$. 